\title{
Federico Birabén e sua proposta para a oficina bibliográfica brasileira. Aproximações e distanciamentos da documentação na América do Sul
}

\author{
Carlos Henrique Juvêncio* \\ Georgete Medleg Rodrigues*
}

Artículo recibido:

25 de mayo de 2015

Artículo aceptado:

27 de septiembre de 2016

\section{Resumen}

En el ámbito del proyecto Repertorio Bibliográfico Universal, ideado y creado por Paul Otlet y Henri La Fontaine, personas e instituciones fueron llamadas para difundir los ideales del dúo belga en todo el mundo. Una de esas personas fue Federico Birabén, ingeniero y bibliotecario argentino responsable por la instalación de las oficinas bibliográficas en Argentina, Chile y Perú bajo la orientación de la propuesta del Instituto Internacional de Bibliografía. En 1910 Birabén propone la creación de la Oficina Bibliográfica Brasileña, siendo un dato investigado en este trabajo que abunda sobre la historia del personaje y acerca de su proposición bibliográfica en Brasil.

* Universidade de Brasília, Brasil

carloshjuvsilva@yahoo.com.br georgete@unb.br

INVESTIGACIÓN BIBLIOTECOLÓGICA, vol. 32, núm. 75, abril/junio, 2018, México, ISSN: 2448-8321 pp. 69-82 
Para ese reto fueron utilizados documentos archivísticos de la época y se realizó una revisión de literatura acerca del desempeño del ingeniero. El artículo concluye que, de hecho, Birabén busca fomentar la creación de una Oficina Bibliográfica en Brasil de acuerdo con las directrices establecidas por el Instituto de Bruselas y similares a la adoptada en otros países, pero el ingeniero no había tomado conocimiento de la construcción del Servicio de Bibliographia y Documentación en la Biblioteca Nacional de Brasil, algo que contribuyó a dejar de lado aquel proyecto en la ocasión que se destacó el trabajo de Manoel da Silva Cícero Peregrino da Silva, entonces director de la biblioteca.

Palabras clave: Instituto Internacional de Bibliografía; Federico Birabén; Oficina Bibliográfica; Repertorio Bibliográfico Universal

Federico Birabén and his proposal to create a Brazilian bibliographic office: Approaches to and differences in Documentation in South America Carlos Henrique-Juvêncio and Georgete Medleg-Rodrigues

\section{Abstract}

In the context of project led by Paul Otlet and Henri La Fontaine for the creation of the Universal Bibliographic Repertory, individuals and institutions were invited to spread the ideals of the Belgian duo around the world. One such person is Argentine engineer and librarian Federico Birabén, who was responsible for the installation of bibliographical offices in Argentina, Chile and Peru in compliance with the proposals of the International Institute of Bibliography. Birabén proposed the creation of the Brazilian Bibliographic Office in 1910. This paper examines his background and his proposal. To this end, archival documents and texts from the time comprise the scope of our research, which includes a literature review regarding the engineer's actions. The article concludes that Birabén, in fact, sought to encourage the creation of a bibliographic office in Brazil in accord with the guidelines provided by the Brussels Institute and other analogous guidelines adopted in other countries. The 
engineer did not know; however, that construction of the Bibliography and Documentation Service of the National Library was already under way, which resulted in this project being shelved as the Library Director Manoel Cicero Peregrino da Silvás project stepped to the fore.

Keywords: International Institute of Bibliography; Federico Birabén; Universal Bibliographic Repertory; Bibliographic Office

\section{INTRODUÇÃO}

Repertório Bibliográfico Universal (RBU), conjunto documental proclamado patrimônio mundial pela UNESCO ("Le Mundaneum inscrit...," 2014), visava reunir num imenso catálogo/inventário mundial a representação, segundo Otlet (1908), de todas as produções frutos do espírito humano, um grande repositório do saber que visava a disseminação do conhecimento acumulado ao longo dos séculos por meio das técnicas documentalistas (Rayward, 1975).

Diversas instituições ao redor do mundo tomaram parte nesse projeto de Paul Otlet e Henri La Fontaine que, por meio do Instituto Internacional de Bibliografia (IIB), fundado pela dupla em 1895, na cidade de Bruxelas, na Bélgica, conclamavam instituições e governos a se tornarem consorte em tal empreitada. As técnicas empreendidas na descrição, ordenação e representação dos diversos tipos de documentos derivavam, em sua maioria, das práticas da Bibliografia e da Biblioteconomia, dando origem a um novo ramo do saber, a Documentação. Assim:

Os objetivos da Documentação consistem em poder oferecer sobre toda a sorte de fatos e conhecimentos as informações documentadas: $1^{\circ}$ universais quanto ao objeto; $2^{\circ}$ confiáveis e verdadeiras; $3^{\circ}$ completas; $4^{\circ}$ rápidas; $5^{\circ}$ atualizadas; $6^{\circ}$ facéis de obter; $7^{\circ}$ reunidas previamente e prontas para serem comunicadas; $8^{\circ}$ disponibilizadas a um grande público.

A Documentação se estabelece nos dias de hoje sob a base da racionalização e da organização dos vários elementos apoiando-se sobre os dados mais avançados da ciência e da técnica por um lado, do trabalho intelectual e da industrialização por outro. Estabalecer o contato 'entre a elaboração do pensamento e o registro dos conhecimentos'. (Otlet, 1934: 373 bis) 
O Brasil, por meio de seu embaixador em Bruxelas, Oliveira Lima, recebe, em agosto de 1910, carta convidando-o a conhecer e participar do projeto de criação do RBU,bem como incentivando a criação de uma oficina bibliográfica no país visando estabelecer contato permanente com o IIB (Lima, 1910).

De fato, tal ideia não era nova no país, em 1900, Freire (1901) já alertara que o Brasil precisava aderir aos ideais disseminados pelo Instituto de Bruxelas, observa ele que:

O Office [Instituto Internacional de Bibliografia] tem fornecido e continua a fornecer duplicatas do repertorio existentes a varias instituições; d'elle tira egualmente extractos referentes a questões especiaes, mediante insignificante retribuição.

Em resumo, a obra colossal e utilitaria que o Instituto tomou a seu cargo, acha-se em plena elaboração.

Para a completa realisação do seu programma, dirige o Instituto um appello aos trabalhadores intellectuaes de todos os paizes.

Podemos nós, brasileiros, recusar-lhe a nossa cooperação?

$\mathrm{Na}$ America, depois dos Estados-Unidos, cuja parte em todo o movimento foi vista no decorrer da exposição, o Governo Mexicano, comprehendendo todo o interesse que havia no desenvolvimento da obra encetada, creou o Instituto Bibliographico Mejicano, instituição annexa á Bibliotheca Nacional, e cuja missão é reunir os elementos para a bibliographia geral do Mexico [...]

Realisar um tal objectivo equivale a collaborar para seu desenvolvimento, fortalecendo ao mesmo tempo o espirito de nacionalidade.

Portanto, sob este ponto de vista, não temos o direito de regatear no nosso auxilio á obra commum; cumpre-nos o dever de contribuir para o patrimonio da humanidade com a Bibliographia Brazileira. $\left(\right.$ Freire, 1901: 155) ${ }^{1}$

Freire demonstra sua afinidade com a causa de Otlet e La Fontaine, vislumbrando a criação de fontes de informações como um meio para o desenvolvimento e o progresso das nações. Porém, apesar de seu apelo, uma década após a publicação de seu texto o Brasil ainda não contava com uma oficina bibliográfica, até que em agosto de 1910, desembarca no Rio de Janeiro para promover o Congreso Sudamericano de Ferrocarriles, o engenheiro argentino Federico Birabén, um dos grandes promotores e difusores dos ideais de Otlet e La Fontaine na América do Sul (Suárez, 1980).

Nesse sentido, este artigo busca investigar um pouco da história de tal personagem bem como trazer à tona a sua proposta para a criação de uma oficina bibliográfica brasileira nos moldes daquelas que ajudou a implementar na Argentina, Chile e Peru. 


\section{As ofiCINAS BIBLIOgRÁfICAS ARgENTINA, CHILENA E PERUANA}

Federico Birabén, engenheiro civil e bibliotecário nascido na cidade de Montevidéu (Uruguai) em 1867, faleceu em Buenos Aires (Argentina) em 1929 e é reconhecido como um dos maiores divulgadores da causa internacionalista bibliográfica, estando à frente ou ajudando a criar instituições com esta filosofia na Argentina, no Chile e no Peru (Suárez, 1980; Romanos de Tiratel, 2004). Diversas obras abordam o trabalho do personagem, bem como sua atuação é destaque no Bulletin de L'Institut International de Bibliographie, que o posiciona como um dos maiores incentivadores do projeto do IIB na América Latina. ${ }^{2}$

Romanos de Tiratel (2004:5-6) diz que:

[...] Este engenheiro e bibliotecário [Birabén] apresenta em Santigo do Chile, no fim de 1908, uma comunicação no IV Congresso Científico Latino-americano e I Pan-americano, onde propõe a criação do Escritório Bibliográfico Nacional como um centro "destinado a dar satisfação às exigências já em voga por conta de nosso avanço intelectual” e que se agrega à "tripla consideração de se tratar de um congresso científico, internacional e americano, me parece razão suficiente para submeter uma questão que afeta em alto grau o conhecimento científico, de âmbito internacional, e, finalmente, que envolve um inventário do progresso real para a maior parte, talvez, dos países americanos”. Se declara seguidor dos ideais e da classificação adotada pelo Instituto Internacional de Bibliografia de Bruxelas e que propôs "a solução do problema bibliográfico nacional". Finalmente, considera que a realização de seu projeto escapa da esfera privada e deve contar com o apoio estatal (Birabén, 1908). Em suma: a necessidade de identificar e disseminar a produção intelectual que só aumenta, o internacionalismo e pan-americanismo, a adoção de normas de descrição bibliográfica e de classificações que possibilitem a cooperação entre os diferentes países, e o reconhecimento de que este tipo de projeto só será concretizado adequadamente com a intervenção oficial, são características marcantes do pensamento de Birabén que, entre março de 1909 e maio de 1910, ajuda na criação de três oficinas bibliográficas: no Chile, na Argentina e no Peru.

2 Segundo o Bulletin de L'Institut International de Bibliographie (1908), o Instituto contava com os seguintes correspondentes sul-americanos interessados na Documentação: "Brésil (81). Bibliothèque : Biblioteca nacional, Rio de Janeiro. - Périodique: Annaes da Biblioteca nacional. - Bibliographie: Revista Sul Americana (contenant la Bibliographie courant du Brésil), Rio de Janeiro; Argentine (82). - Bibliothèque: Biblioteca nacional, Buenos-Ayres; Chili (83). - Bibliothèque: Biblioteca nacional, Santiago de Chile. - Bibliographie: Annuario de la Prensa Chilena; Bolivie (84). - Bibliothèque: Biblioteca nacional, La Plata; Pérou (85). - Bibliothèque: Biblioteca nacional, Lima; Vénézuela (87). - Bibliothèque : Bibliothèque de l'Université centrale, Caracas. - Bibliographie : Bibliographie nationale organisée par le Bureau général de la Statistique ; Uruguay (891). - Bibliothèque : Biblioteca nacional, Montevideo. - Bibliographie : Boletin bibliographico Uruguayo, Montevideo” (Bulletin, 1908). 
Nesse sentido, Birabén advoga em prol da organização universal segundo o modelo proposto por Otlet e La Fontaine, para ele a coordenação dos trabalhos bibliográficos não só ajudará no desenvolvimento latino-americano, bem como ajudará na paz perpétua entre as nações (Suárez, 1980: 11-12), cerne da causa internacionalista divulgada pelos juristas belgas.

Sob este prisma, Menéndez Navarro, Olagüe de Ros e Astrain Gallart (2002) destacam que os esforços para a participação nos ideais do Instituto Internacional de Bibliografia, sobretudo por parte dos países mais desenvolvidos do continente (Brasil, Argentina, México e Chile), tinham relação estreita com a vontade de fortalecimento da comunidade científica nacional destes.

Birabén prestou atenção às considerações metodológicas da ciência documental, detendo sua atenção a função uniformizadora universal desempenhada pela CDU. Suas reflexões doutrinares se completavam com a apresentação dos trabalhos e objetivos do Instituto de Bruxelas e do Repertório Bibliográfico Universal, e a adesão aos princípios internacionalistas e federalistas que inspiravam tais projetos. (Menéndez Navarro, Olagüe de Ros e Astrain Gallart, 2002: 227)

Birabén encontrou terreno fértil para a realização de sua empreitada na biblioteca pública argentina, sob a égide de sua Comissão Protetora, onde passa a advogar pela causa Documentalista e busca formar oficinas bibliográficas que visassem a divulgação de informações por meio das bibliografias (Menéndez Navarro, Olagüe de Ros e Astrain Gallart, 2002). Desta forma, após árdua negociação com o governo argentino e grande promoção dos ideais do IIB naquele país, Birabén consegue que a Oficina Bibliográfica Nacional seja inaugurada em 10 de novembro de 1909. Entre suas atribuições, destacamos o fato desta se responsabilizar pela criação do catálogo coletivo nacional e pela edição da bibliografia nacional, em consonância com os ideais difundidos pelo Instituto Internacional de Bibliografia e seguindo os preceitos por eles estabelecidos para a descrição documental (Suárez, 1980).

Em ação semelhante, onde a propaganda junto ao governo e a instituições governamentais foi intensa, Birabén já havia ajudado a fundar, em 09 de março de 1909, a Oficina Bibliográfica Chilena. Por fim, em 31 de maio de 1910, ajuda na fundação da Oficina Bibliográfica Peruana (Suárez, 1980).

A primeira das oficinas bibliográficas nacionais a se constituir foi a chilena, cujo decreto de criação é de março de 1909. Como já vimos em outro trabalho, a Biblioteca Nacional do país andino tinha permutas bibliográficas com o Instituto de Bruxelas desde 1895 [...]. Junto ao decreto de 
Criação da Oficina, Huneuus ${ }^{3}$ promoveu a contratação temporária de Birabén "para formar o pessoal e colocar a Oficina em condições de funcionar com seus próprios elementos para a satisfação do governo chileno”. A Oficina, cujas atividades reais pouco podemos averiguar, foi, finalmente, anexada à Biblioteca Nacional do Chile.

[...]

A Oficina Bibliográfica Nacional do Peru foi criada mediante um decreto do Ministério da Justiça e Instrução Pública de 31 de março de 1910, com o funcionamento e organograma análogos ao das oficinas chilena e argentina. O decreto anexa a Oficina à Universidad Mayor de San Marcos de Lima, e contemplou a assistência e ajuda da oficina homóloga argentina para sua organização e colocação em funcionamento. A visita de Birabén à Lima se completou com a elaboração de um projeto de base para uma Bibliografia Peruana de Engenharia, que contemplava a criação de um repertório retrospectivo e corrente das publicações peruanas de tal especialidade. A elaboração da bibliografia, confeccionada de acordo com as diretrizes de Bruxelas, foi incumbida a Sociedad de Ingenieros del Perú, cujo Boletim deveria trazê-la à luz, como anexo, além de uma edição independente e em fichas soltas. (Menéndez Navarro, Olagüe de Ros e Astrain Gallart, 2002: 237-238)

Assim, em pouco menos de um ano, Birabén se firma como líder do movimento bibliográfico-documentalista na América do Sul. Ajudando a divulgar os ideais de Otlet e La Fontaine no continente, fomentando a criação de bibliografias nacionais e a difusão do conhecimento. Ademais, outra contribuição do engenheiro foi a idealização de um curso de Bibliografia na Biblioteca Nacional argentina, difundindo, assim, as técnicas documentalistas e bibliográficas utilizadas na criação e manutenção do RBU.

\section{BIRABÉN E SUA PROPOSTA PARA O BRASIL: \\ O CLUB DE ENGENHARIA COMO PALCO}

Consolidando três projetos em três países distintos, Birabén volta sua atenção ao Brasil. Assim, em visita ao país no mês de agosto ${ }^{4}$ de 1910 , com vistas a divulgar o Congreso Sudamericano de Ferrocarriles (Suárez, 1980: 12), o engenheiro aproveitou para realizar, no dia 26 de agosto, palestra sobre o

3 Ministro Chileno da Instrução Pública.

4 Segundos nos fala o Correio Paulistano, Federico Birabén permaneceu no Rio, aproximadamente, entre os dias 19 de agosto de 1910 e o dia 3 de setembro do mesmo ano. Indo embarcar no Porto de Santos (SP) para Buenos Aires (Correio Paulistano, 1910). 
tema "Bibliographia Internacional", 5 na Escola Politécnica do Rio de Janeiro (O Paiz, 1910). À época, o jornal Correio Paulistano (1910: 1) publicou que:

O dr. Biraben, além dessa incumbencia official [o Congresso ferroviário] , occupa-se de um outro assumpto de grande importancia - a creação de repertições bibliographicas internacionaes, segundo um projecto de sua lavra, que já mereceu do Congresso Scientifico Internacional, reunido em junho em Buenos Aires, um voto no sentido de ser recomendado á Conferencia Americana.

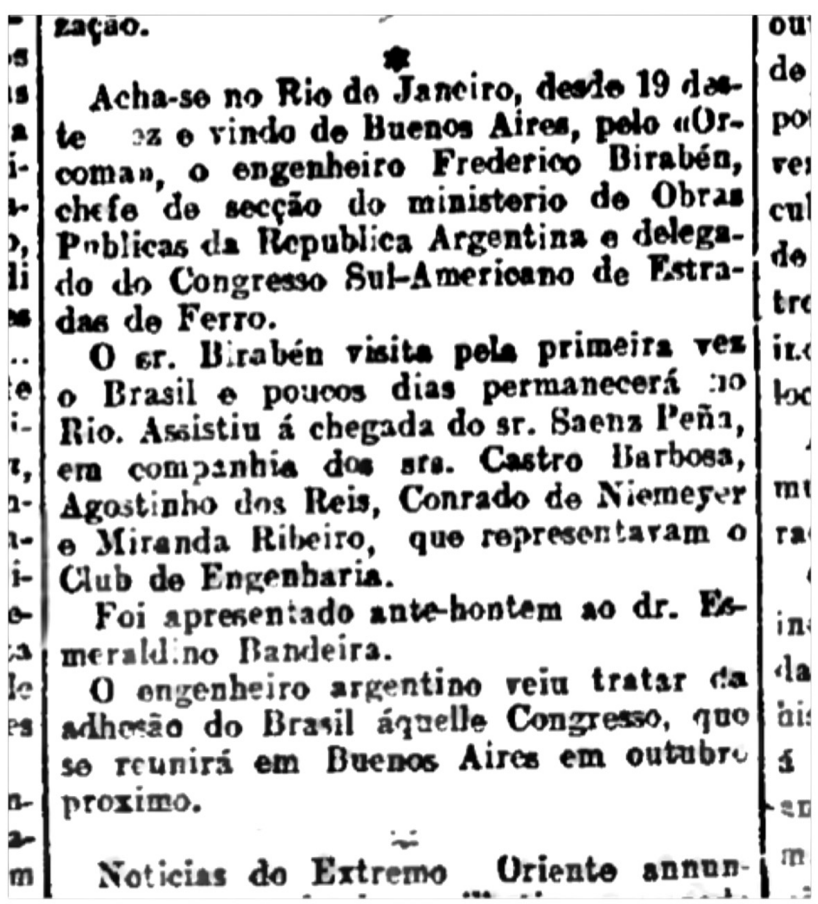

Figura 1. Datalhe do jornal Correio Paulistano de 22 de agosto de 1910

Menéndez Navarro, Olagüe de Ros e Astrain Gallart (2002: 238-239) destacam o acontecimento e observam que:

O trabalho de propaganda de Birabén não se encerra nos meses seguintes [à fundação das oficinas bibliográficas citadas anterioremente], ele volta seu foco para o Brasil com vistas a estimular a participação de autoridades e 
engenheiros brasileiros no Congresso Ferroviário Sul-americano que aconteceria em Buenos Aires no final de 1910 e do qual Birabén foi secretário. Embora não tenhamos nenhuma evidência documental, um de seus discípulos, Pedro B. Franco, aponta a apresentação de um projeto de criação da Oficina Bibliográfica Nacional Brasileira [sic], assim como a adoção da CDU nas diversas bibliotecas do país. Nesse mesmo sentido apontam outras informações indiretas, que confirmam que o citado projeto foi favoravelmente acolhido e publicado em agosto de 1910 no Diário Oficial do Brasil. ${ }^{6}$ Finalmente, em 1915, se estabelece a Oficina Bibliográfica Nacional Brasileira. ${ }^{7}$

Interessante notar nesta fala, que os autores atribuem a criação da dita "Oficina Bibliográfica Brasileira” à atuação de Birabén. Entretanto, nossa pesquisa aponta numa direção distinta a essa. Contudo, antes de aprofundarmo-nos nesta reflexão, esmiuçaremos a proposta de Birabén para o Brasil, publicada no dia 29 de agosto de 1910 pelo Correio Paulistano, sob o título de Assumptos Bibliographicos. A proposta diz respeito à criação de um "Projecto de base para um gabinete (officina) bibliographico nacional"; um "Projecto das bases para uma Bibliotheca Brasileira de Engenharia”; e de um projeto base "[...] para um curso livre de bibliographia technica" ("Assumptos Bibliographicos”, 1910).

Nesse sentido, nos ateremos ao primeiro e ao último projeto, tendo em vista que o segundo demandaria uma pesquisa mais extensa e fora do escopo deste artigo. Isso posto, cabe ressaltar que o projeto para a criação de um gabinete bibliográfico nacional contemplava a criação do catálogo coletivo nacional; a criação da bibliografia nacional; a cooperação com a bibliografia universal; os estudos das questões e problemas bibliográficos e a difusão de tal conhecimento; e o incentivo à unificação dos métodos bibliográficos utilizados nas bibliotecas do país, transformando-as em serviços de documentação ("Assumptos Bibliographicos”, 1910).

Com relação à proposta de um curso livre de bibliografia, este visava "proporcionar aos que o fizerem os conhecimentos e pratica especiaes requeridos para a organização e serviço das bibliothecas technicas e para as tarefas

6 A publicação no Diário Official ocorre em 31 de agosto de 1910, sob o título "Bases para una Oficina Bibliográfica Nacional Brasileira, presentadas al Exm. Sr. Ministro del Interior, Dr. Esmeraldino Bandeira, por el ing. Frederico Birabén, director de la O. B. N. argentina”. Contudo, isso não constitui na autorização para que a mesma fosse instalada no país, sendo apenas o conteúdo da apresentação - um caso semelhante ao publicado no Correio Paulistano, conforme veremos à frente. A publicação traz ainda os projetos das oficinas argentina, chilena e peruana.

7 Juvêncio (2014) observa que não se sabe ao certo sobre qual projeto os autores se referem, mas eles não dizem respeito ao da Biblioteca Nacional, que se concretiza em 1911 com a criação do Serviço de Bibliographia e Documentação. O autor ainda nos alerta que, talvez, esta citação faça referência ao projeto de Oficina Bibliográfica no Club de Engenharia, mas somente uma pesquisa aprofundada poderá sanar tal dúvida. 
de bibliographia em geral" ("Assumptos Bibliographicos", 1910: 4). Ou seja, preparava pessoal para trabalhar com a CDU e aplicar as técnicas bibliográficas e documentalistas no ambiente em que atuassem.

Não muito distante dos projetos já implementados na Argentina, no Chile e no Peru, ${ }^{8}$ constando com poucas modificações no caso brasileiro, o objetivo primordial da proposta de Birabén seria a construção de uma rede de informações na América Latina, buscando concatenar os países onde havia atuado para que participassem do projeto do IIB.

Contudo, ao menos no Brasil, Birabén parecia não estar a par do que se estava construindo em termos de Bibliografia e Documentação, tendo em vista que o projeto bibliográfico idealizado por Manoel Cícero Peregrino da Silva (então diretor da Biblioteca Nacional) remontava a 1902, onde este propõe no projeto de novo regulamento da instituição que se crie o:

\section{[...] Instituto Bibliographico Brasileiro}

Art. 115. É creado e funccionará annexo á Bibliotheca Nacional o Instituto Bibliographico Brasileiro, cujos fins serão: $1^{\circ}$ organisar por meio de cartões e segundo o systema de classificação decimal um repertorio bibliographico, como contribuição brasileira para a constituição da bibliographia universal, abrangendo as obras de auctores nacionaes ou estrangeiros impressas ou editadas no paiz, as de auctores nacionaes impressos no estrangeiro ou ineditas e as de auctores estrangeiros que se occupem especialmente do Brasil, comprehendidos os artigos de periodicos e escriptos de qualquer natureza; $2^{\circ}$ adquirir para expor ao exame dos estudiosos, como fará com o repertorio brasileiro, uma duplicata dos repertorios estrangeiros que estiverem organisados e se forem organizando. (Silva, 1902).

Tal instituição, após quase uma década de debate e negociações, é criada em 1911, mas não como o instituto independente denotado na proposta de 1902, e sim como um dos serviços da Biblioteca Nacional, o Serviço de Bibliographia e Documentação:

Art. 137. O serviço de bibliographia e documentação, em correspondencia com o do Instituto Internacional de Bibliographia de Bruxellas, abrangerá: $1^{\circ}$, a organização, segundo o systema de classificação decimal e por meio de fichas, do repertorio bibliographico brasileiro como contribuição para o repertorio bibliographico universal, de modo a comprehender as obras de autores nacionaes ou estrangeiros, impressas ou editadas no paiz, as de autores nacionaes, impressas no estrangeiro ou ineditas e as de autores estrangeiras 
que se occuparem especialmente do Brazil, incluidos os artigos insertos em publicações periodicas e os escriptos de qualquer natureza;

$2^{\circ}$, a impressão dessas fichas para serem expostas á venda ou permutadas por fichas de repertorios estrangeiros;

$3^{\circ}$, a acquisição de um exemplar de cada uma das fichas que constituem os repertorios estrangeiros, já organizados e que se forem organizando;

$4^{\circ}$, a cooperação da Bibliotheca na organização do repertorio-encyclopedico universal;

$5^{\circ}$, a organização do catalogo collectivo das bibliothecas brazileiras;

$6^{\circ}$, o uso publico dos repertorios e do catalogo collectivo. (Brasil. Decreto $8.835,1911)$

De fato, podemos observar já no primeiro artigo a correspondência entre o Serviço brasileiro e o Instituto belga, sobretudo por seus ideais serem um reflexo direto daqueles apregoados pela dupla belga e, de certa maneira, àqueles difundidos por Birabén. Além de tal fato, Peregrino escreve ao secretário do IIB, Louis Masure, comentando sobre a criação do Serviço, declarando sua inspiração nos ideais da instituição, observa ele que: "As disposições que compõem o capítulo sobre a Bibliografia e a Documentação [no Regulamento de 1911] são um pouco do mesmo projeto que organizei por ocasião da visita ao vosso Instituto e que comuniquei ao senhor Birabén quando ele esteve no Rio de Janeiro" (Silva, 1911). ${ }^{9}$

Nesse sentido, parece deixar claro que a proposta brasileira foi elaborada em 1907, por ocasião da visita do então diretor da BN ao Instituto, ${ }^{10}$ ou seja, cerca de 3 anos antes de Birabén desembarcar no Rio de Janeiro. Além disso, Peregrino, ao que tudo indica, expôs ao engenheiro argentino que já havia uma proposta de criação de uma oficina bibliográfica brasileira. Decerto, a proposta de Birabén e Peregrino da Silva se aproximam, ambas denotam a vontade de se construir a Bibliografia Nacional de acordo com os preceitos do IIB, bem como visam colaborar com o RBU.

De fato, Otlet e La Fontaine (1895) formulam os objetivos do RBU da seguinte maneira: $1^{\circ}$ ) O RBU deve ser completo, compreendendo a bibliografia do passado e do presente; $2^{\circ}$ ) A ordem do repertório deve ser tanto ideológica como onomástica, ou seja, tanto por assunto, quanto por autor; $3^{\circ}$ ) Como instrumento de pesquisa, o RBU deve estar disponível em todos os centros intelectuais; $4^{\circ}$ ) O RBU deve ser exato e preciso, mas deve também permitir a sua correção de modo simples e rápido; $\left.5^{\circ}\right) \mathrm{O}$ repertório

9 "Les dispositions qui constituent le chapitre concernant la bibliographie et la documentation sont a peu pers les mêmes du projet que j'avais organisé arres ma visite a votre Institut et que j'ai communiquêes a monsieur Biraben quand il a été a Rio de Janeiro.”

10 Para maiores informações consultar Juvêncio (2014). 
deve estar totalmente disponível ao público; $6^{\circ}$ ) O RBU deve estar associado a uma rede de catálogos de bibliotecas, permitindo o acesso rápido às obras; $7^{\circ}$ ) A iniciativa deve servir de estatística intelectual acerca das obras produzidas pelo espírito humano; $8^{\circ}$ ) O repertório deve assegurar aos autores a proteção legal de suas obras intelectuais.

Desta forma, ao observarmos as propostas tanto de Birabén como de Peregrino, podemos concluir que ambas são reflexos dos ideais de Paul Otlet e Henri La Fontaine, sobretudo, por incentivarem a criação de bibliografias nacionais e de redes de bibliotecas, além de incentivarem a construção do Repertório Bibliográfico Universal.

\section{Conclusão}

Ao longo deste artigo, podemos perceber que as propostas de Birabén e Peregrino da Silva possuem muito mais afinidades do que distanciamentos, de fato, ambas baseiam-se no ideal apregoado pelo Instituto de Bruxelas. Contudo, parece-nos claro que o personalismo de cada um dos personagens busca a aproximação direta com o Instituto Internacional de Bibliografia. Além do mais, apesar das inúmeras aproximações ideológicas entre Peregrino da Silva e o engenheiro argentino, a proposta brasileira se inseria num contexto de mudanças profundas no fazer biblioteconômico na Biblioteca Nacional. Um exemplo disso é que, ao invés de um curso livre de Bibliografia, a BN funda um curso de Biblioteconomia, que contemplava esta disciplina como uma de suas áreas do saber (Silva, 1911). Nesse sentido, mais do que as técnicas bibliográficas, Peregrino da Silva parecia buscar o desenvolvimento de um curso que formasse bibliotecários. Outro exemplo é que a construção da bibliografia brasileira estava prevista em outro instrumento legislativo, a Lei de Depósito Legal (Brasil. Decreto 1.825, 1907), que previa a edição de tal publicação como uma forma de controle bibliográfico da produção editorial nacional.

Deste modo, podemos concluir que, apesar de Peregrino da Silva optar pelo personalismo na construção de seu projeto, ambos são fruto dos ideais propagados pelo Instituto Internacional de Bibliografia e buscavam tomar parte na construção do Repertório Bibliográfico Universal, bem como na disseminação das técnicas documentalistas e bibliográficas. 


\section{REFERENCIAS}

"Assumptos Bibliographicos". 1910. Correio Paulistano, 29 ago. Acesso em: 15 nov. 2013. http:/www.arquivoestado.sp.gov.br/hemeroteca/exemplares.php?idTituloExemplar $=9292$

Brasil. Decreto 8.835, de 11 de julho de 1911: Approva o regulamento da Bibliotheca Nacional. Diário Oficial da União, 16 jul. 1911. Acesso em: 23 maio 2012. http:// www2.camara.leg.br/legin/fed/decret/1910-1919/decreto-8835-11-julho-1911502890-republicacao-102224-pe.html

Brasil. Decreto 1.825, de 20 de dezembro de 1907: Dispõe sobre a remessa de obras impressas á Bibliotheca Nacional. Diário Oficial da União, 21 dez. 1907. Acesso em: 23 maio 2012. http://www2.camara.leg.br/legin/fed/decret/1900-1909/decreto-1825-20-dezembro-1907-509239-publicacaooriginal-1-pe.html

Bulletin de L'institut International de Bibliographie. 1908. Ano 3.

Correio Paulistano. 1910. 24 ago. Acesso em: 15 nov. 2013. http://www.arquivoestado.sp.gov.br/hemeroteca/exemplares.php?idTituloExemplar $=9292$

Freire, Victor da Silva. 1901. "A Bibliographia universal e a Classificação decimal". Annuario da Escola Polytechnica de São Paulo 2: 125-157.

Juvêncio, Carlos Henrique. 2014. "O Mundaneum no Brasil: o Serviço de Bibliographia e Documentação da Biblioteca Nacional e seu papel na implementação de uma rede de informações científicas”. Dissertação, Mestrado em Ciência da Informação, Faculdade de Ciência da Informação, Universidade de Brasília.

"Le Mundaneum inscrit officiellement à l'Unesco pour son «Google de papier»: 4ème reconnaissance pour Mons”. 2014. La Province, 8 maio 2014. Acesso em: 10 maio 2014. www.laprovince.be/1002208/article/2014-05-08/le-mundaneum-inscrit-officiellement-a-1-unesco-pour-son-google-de-papier-4eme-re

Lima, Oliveira. 1910. Ofício ao ministro das Relações Exteriores, Barão do Rio Branco. Bruxelas, 23 ago. (Arquivo Histórico do Itamarty, 205,1,03).

Menéndez Navarro, Alfredo, Guillermo Olagüe de Ros e Mikel Astrain Gallart. 2002. "Ciencia, positivismo e identidad nacional en el Cono Sur: la participación argentina en los proyectos documentales contemporáneos (1895-1928)”. Hispania 62 (210): 221-258.

O Paiz, 26 ago. 1910. Acesso em: 15 nov. 2013. http://memoria.bn.br/DOCREADER/ docmulti.aspx? $\mathrm{BIB}=178691$

Otlet, P. 1934. Traité de Documentation: le livre sur le livre, théorie et pratique. Bruxelas: Mundaneum. pp. 373 bis.

Otlet, Paul e Henri La Fontaine. 1895. "Création d’un Répertoire Bibliographique Universel: note préliminaire”. Bulletin de L'Institut International de Bibliographie $1(1): 15-38$.

Otlet, Paul. 1908. "L’Office International de Bibliographie”, 358-374. Le Mouvement scientifique en Belgique: 1830-1905, t. II. Bruxelas: Société Belge de Librairie. Rayward, W. Boyd. 1975. The universe of information: the work of Paul Otlet for Documentation and international organisation. Moscow: VINITI, FID.

Rayward, W. B. 1975. The universe of information: the work of Paul Otlet for Documentation and international organisation. Moscow: VINITI; FID. 
Romanos de Tiratel, Susana. 2004. "La bibliografía nacional argentina: una deuda pendiente", em World Library and Information Congress: IFLA General Conference and Concil 70. Buenos Aires, Anais... Buenos Aires: IFLA.

Silva, Manoel Cícero Peregrino da. 1902. Projecto de Regulamento para a Bibliotheca Nacional do Rio de Janeiro. Rio de Janeiro.

Silva, M. C. P. 1911. Carta ao secretário do Instituto Internacional de Bibliografia. Rio de Janeiro, 28 nov. (Biblioteca Nacional, Mss 69,4,010).

Suárez, Reinaldo José. 1980. "Biraben, precursor de la Classificación Decimal y de la enseñanza bibliotecária”. Boletín Bibliotecológico de La Plata 1.

Para citar este texto:

Juvêncio, Carlos Henrique y Georgete Medleg Rodrigues. 2018.

"Federico Birabén e sua proposta para a oficina bibliográfica

brasileira. Aproximaçãoes e distanciamentos da documen-

tação na América do Sul". Investigación Bibliotecológica: archi-

vonomía, bibliotecología e información 32 (75): 69-82.

http://dx.doi.org/10.22201/iibi.24488321xe.2018.75.57944 